\title{
Hybrid Electrical Energy Storage Systems
}

\author{
Massoud Pedram ${ }^{\dagger}$, Naehyuck Chang", Younghyun Kim», and Yanzhi Wang \\ University of Southern California, CA, USA \\ $*$ Seoul National University, Korea \\ †\{pedram, yanzhiwa\}@usc.edu, $¥\{$ naehyuck, yhkim\}@elpl.snu.ac.kr
}

\begin{abstract}
Electrical energy is a high quality form of energy that can be easily converted to other forms of energy with high efficiency and, even more importantly, it can be used to control lower grades of energy quality with ease. However, building a cost-effective electrical energy storage (EES) system is a challenging task despite steady advances in the design and manufacturing of EES elements including various battery and supercapacitor technologies. As of today, no single type of EES element fulfills high energy density, high power delivery capacity, low cost per unit of storage, long cycle life, low leakage, and so on at the same time.

Unlike conventional EES systems, we introduce a HEES (hybrid EES) system comprising heterogeneous EES elements. Our proposed HEES system builds on the concepts of computer memory system architecture and management in order to achieve the attributes of an ideal EES system through appropriate allocation and organization of various types of EES elements. We also introduce a HEES design considerations which should be taken into account to optimize the amortized cost for the system, including the initial cost (cost per capacity), the operating cost (efficiency), the maintenance cost (cycle life and disposal cost), and so forth.
\end{abstract}

\section{Categories and Subject Descriptors}

$$
\text { B.0 [General] }
$$

\section{General Terms}

Design

\section{Keywords}

Energy, Energy storage, Electrical storage, Hybrid storage, Charge, Management

\section{INTRODUCTION}

An electrical energy storage (EES) system is an energy reservoir which can store energy electrically and supply energy when necessary. Generally EES systems perform three functions of energy management, power bridging, and power quality control. The EES is considered to store a higher grade of energy quality than most other storage systems since electrical energy has a greater capacity to feedback and control lower grades of energy quality [1]. The EES systems may supply power to portable electronics (a few Wh or smaller), provide uninterruptible power (hundreds of Wh), and mitigate peak power demand, thereby reducing stress on the Power
Grid (GWh or larger). A typical EES consists of a single type of EES element.

Performance metrics of EES systems include cycle efficiency, cost per unit capacity, energy density, power capacity, cycle life, environmental effect including end-of-life disposal cost. An ideal EES system is one which exhibits the best possible performance with respect to all these metrics so that it will have the minimum amortized (dollar or environmental) cost during its whole lifetime. Unfortunately, no single type of EES element can simultaneously fulfill all the desired characteristics of an ideal EES system, and thus minimize the amortized lifetime cost of EES.

Recalling computer memory hierarchy, no single type of memory device can achieve short access time, high density, balanced read/write performance, low cost per bit, low power consumption, non-volatility, etc. A practical way to provide a cost-effective memory system is construction of a hierarchical memory system. In this paper, we introduce a HEES (hybrid EES) system comprising heterogeneous EES elements in order to take advantage of the strengths of individual element types while hiding their shortcomings in the same way that a computer system builds a highly efficient memory system by using different types of memory elements. The HEES system architecture connects the EES elements via power buses and various power converters to enable charge transfer among the EES elements. It consists of various EES elements that have different characteristics such as cycle efficiency, leakage current, cycle life, storage cost, and volumetric energy density, power rating, and so on.

In this paper, we will present the principles of the HEES, and demonstrate its benefits according to a lifecycle analysis. The proposed HEES system consists of multiple banks of heterogeneous EES elements. Each bank in turn consists of an array of homogeneous EES elements so that the bank may meet certain voltage, current and capacity requirements. As the charging/discharging efficiency of the bank is strongly dependent on the SOC (state of charge) [2,3], dynamic reconfiguration of the array structure may help maintain a higher efficiency over a fixed array size.

Moreover we introduce HEES management polices to minimize the amortized lifetime cost of the system. The management policies include i) charge allocation, which determines the type and amount of EES element used to store electrical energy when an excessive amount of electricity is input to the system, ii) charge migration, which transfers charge (electrical energy) between two EES elements considering the discharge power capacity of the provider as well as the energy storage efficiency of the receiver, and leakage of the EES elements for the long-term storage, and iii) charge replace- 
ment (discharge ${ }^{1}$ ), which determines the type and amount of each EES element needed to meet a particular level of energy demand.

More precisely, the objective of the proposed EES system is to greatly improve the efficiency and reduce the cost of powering large-scale manufacturing and commercial sites by using i) a HEES comprised of different types of energy storage elements organized in an appropriate storage hierarchy and reconfigured on the fly, ii) performance-optimized electronics for online reconfiguration of the storage system and for maximizing the energy conversion and distribution efficiency from the electrical Power Grid and distributed/renewable energy sources to the load, and iii) energy management, load leveling, and peak shaving techniques depending on the power usage profile of the target load and the power availability and cost factors.

\section{ENERGY STORAGE DEVICES}

\subsection{Performance Metrics}

\subsubsection{Capital cost}

Capital cost, which is one of the most important considerations in the design of an EES system, can be represented in the forms of cost per unit of delivered energy $(\$ / \mathrm{kWh})$ or per unit of output power $(\$ / \mathrm{kW})$. Capital cost is an especially important concern when constructing HEES systems. This is because such systems often consist of EES elements with relatively low unit cost (e.g., Lead-acid batteries) and EES elements with relatively high unit cost (e.g., supercapacitors). The overall HEES system cost must be minimized by allocating the appropriate amounts of low-cost vs. high-cost EES elements while meeting some other constraints such as cycle efficiency, total energy storage capacity, or peak output power rate.

\subsubsection{Cycle efficiency}

The cycle efficiency of an EES element is defined by the "roundtrip" efficiency, i.e., the energy input and output efficiency for charging and then discharging. The cycle efficiency is the product of charging efficiency and discharging efficiency, where charging efficiency is the ratio of electrical energy stored in an EES element to the total energy supplied to that element during the entire charging process, and discharging efficiency is the ratio of energy derived from an EES element during the discharging process to the total energy stored in it.

Charging/discharging efficiency can be significantly affected by charging/discharging profiles and ambient conditions. For example, the well-known rate capacity effect dictates that the total charge or energy delivered by a battery exhibiting this effect goes down with the increase in load current, resulting in lower discharging efficiency. Moreover, literature $[4,5]$ tells us that battery efficiency can be enhanced by using pulsed current discharge instead of constant power discharge because of the charge recovery process that happens during the idle time periods in pulsed discharging.

\subsubsection{Cycle life}

The SOH (state of health) of an EES element is a measure of its age. It reflects the general condition of the EES element and its ability to store and deliver energy compared to its initial state (i.e., compared to a fresh new EES element). During the lifetime of the EES element, its capacity or "health" tends to gradually deteriorate due to irreversible physical and chemical changes which take place along with usage until the EES element is no longer usable

\footnotetext{
${ }^{1}$ We use the term 'replacement' to imply discharge so that we use the same term for computer memory management.
}

(the EES element reaches its end-of-life). To indicate the rate at which $\mathrm{SOH}$ is deteriorating, the cycle life may be defined as the number of cycles an EES element can perform before its capacity drops to a specific percentage (60-80\% typically) of its initial fullcharged capacity. It is one of the key performance parameters and gives an indication of the expected working lifetime of the EES element. Obviously cycle life of an EES element is closely related to the replacement period and full cost of the element according to a lifecycle analysis method.

Note that the cycle life of an EES element depends on the depth of discharge (DoD) before the EES element is recharged. For Leadacid batteries, the number of cycles yielded goes up exponentially when lowering the DoD [6]. Similar conclusions hold for most cell chemistries. We shall assume that the EES element is fully charged or discharged during each cycle, unless otherwise specified.

EES elements whose operation principles are largely based on electrical, mechanical or thermal technologies, including capacitor/supercapacitor, flywheel, thermal energy storage (TES) and cryogenic energy storage (CES), typically have long cycle lives. In contrast, cycle life of batteries are not that high due to unavoidable chemical deterioration of the electrodes during their operation. Metal-Air battery currently has the lowest cycle life among commercially available batteries.

\subsubsection{Self-discharge rate}

The self-discharge rate is a measure of how quickly a storage element will lose its energy when it simply sits on the shelf. Determined by the inner structure and chemistry, as well as ambient temperature and humidity, self-discharge characteristics significantly affect the sustainable energy storage period of the given storage element. Generally speaking, Metal-Air batteries have very small self-discharge rate and are thus suitable for long-term storage of energy. Ordinary batteries can store energy reliably up to tens of days. On the other hand, capacitor and supercapacitor have large self-discharge rate compared with others and are useful for shortterm energy storage for a maximum of several hours.

\subsubsection{Power and energy density}

Power density is calculated as the rated output power divided by the volume (or mass) of the EES element. Similarly, energy density is the stored energy divided by the volume (or mass). Among various kinds of storage elements, Metal-Air batteries have the highest energy density (typically $1000 \mathrm{Wh} / \mathrm{kg}$ ). Batteries, TES and CES have medium energy density, while capacitor/supercapacitor and flywheel have the lowest energy density. However, capacitor/supercapacitor and flywheel have very high power densities and are thus suitable for applications with high peak or pulse power requirements. Since a HEES typically takes advantage of the energy density of a primary source for long operational time and the power density of a secondary source for power requirements, a list of power and energy densities of various storage elements may act as guideline for choosing appropriate elements and constructing HEES systems.

\subsection{Commercial Off-the-Shelf Storage Devices}

\subsubsection{Supercapacitor}

Electric double layer capacitors, more commonly known as supercapacitors, are widely exploited to mitigate load current fluctuations in the batteries. Supercapacitors have a superior cycle efficiency which reaches almost $100 \%$, and long cycle life [8]. Moreover, compared with batteries, supercapacitors exhibit significant higher volumetric power density but lower energy density [9]. Thus they are suitable for energy storage in situations with frequent charg- 
Table 1: Comparison between energy storage elements [6, 7].

\begin{tabular}{|c||c|c|c|c|c|c|}
\hline EES Elements & Capital cost $(\$ / \mathrm{kWh})$ & Cycle efficiency & Cycle life & Self-discharge per day & Energy density & Power density \\
\hline \hline Lead-acid & $100-200$ & $70-90 \%$ & $500-800$ & $0.1-0.3 \%$ & $30-50 \mathrm{Wh} / \mathrm{kg}$ & $75-300 \mathrm{~W} / \mathrm{kg}$ \\
\hline NiCd battery & $800-1,000$ & $70-90 \%$ & $2,000-2,500$ & $0.2-0.6 \%$ & $50-75 \mathrm{Wh} / \mathrm{kg}$ & $150-300 \mathrm{~W} / \mathrm{kg}$ \\
\hline NiMH battery & $450-1,000$ & $66 \%$ & $500-1,000$ & $0.5-1 \%$ & $60-80 \mathrm{Wh} / \mathrm{kg}$ & $250-1,000 \mathrm{~W} / \mathrm{kg}$ \\
\hline Li-ion battery & $600-2,500$ & $>90 \%$ & $1,000-10,000+$ & $0.1-0.3 \%$ & $100-250 \mathrm{Wh} / \mathrm{kg}$ & $250-340 \mathrm{~W} / \mathrm{kg}$ \\
\hline NaS battery & $300-500$ & $87 \%$ & 2,500 & $\sim 20 \%$ & $150-240 \mathrm{Wh} / \mathrm{kg}$ & $150-230 \mathrm{~W} / \mathrm{kg}$ \\
\hline Metal-Air battery & $10-60$ & $<50 \%$ & $100-300$ & Very small & $1-10 \mathrm{kWh} / \mathrm{kg}$ & - \\
\hline Supercapacitor & $20,000-50,000$ & $>90 \%$ & $50,000+$ & $20-40 \%$ & $2.5-15 \mathrm{Wh} / \mathrm{kg}$ & $100,000+\mathrm{W} / \mathrm{kg}$ \\
\hline Flywheel & $1,000-5,000$ & $>90 \%$ & $20,000+$ & $100 \%$ & $10-30 \mathrm{Wh} / \mathrm{kg}$ & $400-1,500 \mathrm{~W} / \mathrm{kg}$ \\
\hline High-temperature TES & $30-60$ & $30-60 \%$ & - & $0.05-1 \%$ & $80-200 \mathrm{Wh} / \mathrm{kg}$ & - \\
\hline CES & $3-30$ & $40-50 \%$ & - & $0.5-1 \%$ & $150-250 \mathrm{Wh} / \mathrm{kg}$ & $10-30 \mathrm{~W} / \mathrm{kg}$ \\
\hline
\end{tabular}

ing/discharging cycles or periodic high current pulses. In a batterysupercapacitor hybrid system, the supercapacitor stores surplus energy from the battery during low demand periods, and provides extra energy during peak load current demand period.

However, a distinct disadvantage of a supercapacitor is its large self-discharge rate compared to that of ordinary batteries. A supercapacitor may lose more than $20 \%$ of its stored energy per day even if no load is connected to it. Another important concern of supercapacitors in HEES systems is the terminal voltage variation coming from the characteristics of a capacitor nature whereby the terminal voltage is linearly proportional to its SOC. The terminal voltage increases or decreases accordingly as the supercapacitor is charged or discharged. This terminal voltage variation is much higher than that observed in typical batteries. This effect results in a significant conversion efficiency variation in the power converters which are connected to the supercapacitors. The hybrid energy storage systems including the supercapatior should properly account for the above characteristics to be practical.

\subsubsection{Lead-acid battery}

As one of the oldest and most developed rechargeable battery technologies, Lead-acid batteries have short cycle life (500-800 cycles) and low energy density $(30-50 \mathrm{Wh} / \mathrm{kg}$ ) due to the inherent high density of Lead as a metal. Besides, they also have a poor low temperature performance and thus a thermal management system is required. In spite of these disadvantages, their ability to supply high surge currents means that such Lead-acid cells maintain relative high power density $(75-300 \mathrm{~W} / \mathrm{kg}$ ). These features, along with their low cost (100-200 \$/kWh) and high energy efficiency (70$90 \%$ ), make Lead-acid batteries suitable in motor vehicles so as to provide the high current demand for automobile starter motors. Lead-acid batteries have also been used in a few large-scale commercial energy management systems. The largest one is a $40 \mathrm{MWh}$ system in Chino, California, built in 1988 [10].

\subsubsection{Li-ion battery}

Li-ion battery, first demonstrated in the 1970s, is a family of rechargeable batteries in which lithium ions move from negative electrode to positive electrode through discharging, and in opposite direction during charging. It is now the battery of choice in portable electronic devices and is growing in popularity in military, electric vehicle, and aerospace applications. The growing popularity of Li-ion battery is mainly due to the following reasons: high energy density (100-250 Wh/kg or 250-360 Wh/L) [11, 7], high efficiency (almost 100\%), long cycle life (as high as 10,000 cycles), no memory effect, and low self discharge rate $(0.1-0.3 \%$ per day).

While taking over $50 \%$ of the small portable devices market [7], there are some challenges to build large-scale Li-ion based EES. The main hurdle is the high cost (above $600 \$ / \mathrm{kWh}$ ) due to spe- cial packaging and internal overcharge protection circuits. Several companies, such as SAFT and Mitsubishi, are working to reduce the manufacturing cost of $\mathrm{Li}$-ion batteries to capture large new energy markets, especially markets for the electrical vehicles.

\subsubsection{NiMH batteries}

Nickel-metal hydride battery, abbreviated $\mathrm{NiMH}$, is a type of rechargeable battery similar to the nickel-cadmium cells (NiCd). The only difference is that the former one uses a hydrogen-absorbing alloy for negative electrode instead of cadmium. The energy density of NiMH batteries is more than double that of Lead-acid batteries and $40 \%$ higher than that of NiCd batteries. The NiMH battery relatively inexpensive to purchase. However, they suffer from the memory effect, although much less pronounced than that in the $\mathrm{NiCd}$ ones, and have a rather high self-discharge rate.

The most significant feature of NiMH batteries is the high power density (250-1000 W/kg), which is the highest among existing battery types. Therefore these batteries are widely used in high current drain consumer electronics, such as digital cameras with LCDs and flashlights. Recently, they have been used in hybrid electric vehicles such as the Toyota Prius, Honda Insight, Ford Escape Hybrid, Chevrolet Malibu Hybrid, and Honda Civic Hybrid.

\subsubsection{Metal-Air batteries}

In Metal-Air batteries, the anode is made of pure metal and cathode is connected to an inexhaustible source of air. Thus such batteries can be regarded as "half a fuel cell" [12]. Potentially, MetalAir batteries have the highest energy density (theoretically more than $10 \mathrm{kWh} / \mathrm{kg}$ ) and are relatively inexpensive to produce. They are also environmentally friendly. However, such batteries suffer from the difficulty and low efficiency of the required electrical recharging. Thus instead of offering electrically rechargeable battery, many manufacturers offer refuel units where the consumed metal is replaced mechanically. Therefore Metal-Air batteries are not suitable for EES systems.

\subsubsection{Emerging technologies}

CES [13, 14], which can be regarded as a special type of lowtemperature TES, is a new EES technology. The principle of CES is to utilize the atmosphere as a heat source to vaporize and superheat some cryogenic fluid (e.g., liquid nitrogen or liquid air) in a thermal power cycle. This is different from the conventional heat engines, which use high-temperature heat source and atmosphere as heat sink. The use of liquid nitrogen or liquid air as energy storage medium would not pose any environmental burden. In general, CES has relatively high energy density (150-250 Wh/kg), low capital cost and relatively long storage period, but currently exhibits low efficiency (40-50\%). 


\section{CHARGE MANAGEMENT CIRCUITS}

Charge transfer from a power source, such a Grid, a solar panel, or a windmill, to an EES element mandate voltage and current conversion. Some power sources such as a Grid and an induction generator windmill are AC (alternate current) while EES elements accept DC (direct current). Most batteries require a charging sequence according to their SOC, such as a constant voltage or constant current, which does not allow to accept the all the charge generated from the power source. Charge transfer to and from heterogeneous EES elements require appropriate voltage and current conversion for safety and better cycle efficiency. Even charge transfer between homogeneous EES elements should be carefully managed whenever they have different SOC, which is a common case. Furthermore, transfer charge from an EES element to a power consumer requires proper voltage conversion. Consequently, in general, it is obvious that direct connection between EES elements, power sources and power consumers without a proper power converter may significantly degrades the energy efficiency, or even worse, is highly likely make permanent damage to the elements. We cannot properly control the flow of energy without aid of such charge management electronics.

\subsection{Power Converters and Efficiency}

While some of EES elements have a relatively stable output voltage performance regardless of the operational conditions, others have a varying output voltage according to the load current, SOC, $\mathrm{SOH}$, temperature, and so on. Generally, active control rather than passive control such as the use of resistor should be involved to manage the charge flow direction and amount of charge transfer regardless of the operational condition. Active control requires charging/discharging circuitry to step up/down the voltage operating as a current source or voltage source according to the charge sequence. While EES elements are charged with DC power and also supply DC power, major power provider for the HEES system is the Power Grid, which is an AC power source. Furthermore, almost all power consumers, even though the ultimate power consumer such as VLSI circuit requires DC power, also accept AC power and convert into $\mathrm{DC}$ power later on. This implies that storing and restoring power from and into DC EES elements need AC-DC and DC-AC conversions.

Designing the power converters should be carried out considering not only the initial cost of implementing them, but also the energy cost while operating the system. Low conversion efficiency directly results in a high operational cost. The energy efficiency of the power converters are affected by not only by the design such as switch resistance, inductor and transformer performance, but also by operational conditions such as input voltage, output voltage, load current, switching frequency.

\subsection{Monitoring and Control}

The HEES system should provide a reliable operation. A realtime operating system is necessary to ensure real-time monitoring and control of the system, and some fault-tolerance techniques such as majority voting or redundancy may be applied. The control system should provide a supervisory control for the best energy efficiency across the whole EES system. Automated alarm and sequence control for emergency shut down are also necessary. The control system relies on accurate measurement and monitoring of the each EES element. BMS (battery management system) is one of necessary functions to maintain stability and sources of important information for the charge management policies. It estimates the SOC and SOH, controls the voltage, current, and cell balances (distributing SOC evenly across the bank), and provides diagnostics functionality.

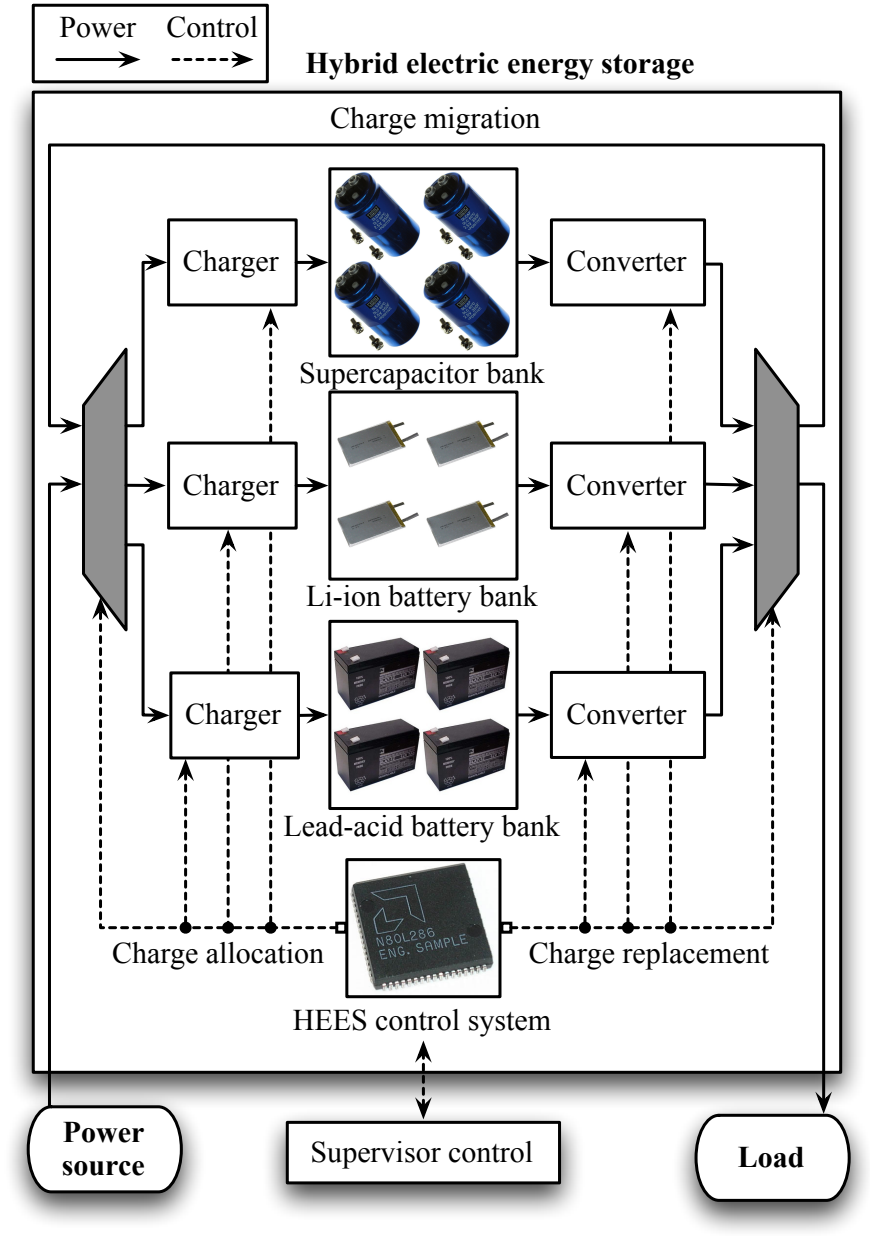

Figure 1: Architecture of the proposed HEES system.

Monitoring the status and controlling each EES element are important for maintaining the HEES system. Due to a large physical dimension and proximity requirement of sensor attachment, single on-board peripheral structure may not be feasible. Thus, distributed sensor boards and control-area network structure is mandatory. Vehicle-area network such as CAN (control-area network) or FlexRay can be good candidates for the remote control systems.

Human interaction should be sometime mandatory for a largescale operation as in a power plant. A large amount of real-time data collection, data logging and visualization are mandatory for system analysis and policy enhancement. Thus, SCADA (supervisory control and data acquisition) system is employed.

\section{HEES SYSTEMS}

\subsection{Architecture}

This paper introduces a HEES architecture, which provides enhanced cycle efficiency, extended cycle life for each EES element, increased energy storage capacity, and output power rating. The architecture is shown in Figure 1. There are heterogeneous EES elements, which are connected with each other through power converters such as a charger and DC-DC converters. As stated in Section 2, each EES element in existence today has its strengths and weaknesses. Once again, there are no single EES elements that fulfill all the requirements of an "ideal" storage system. In this 
context the utilization of heterogeneous EES elements within an energy storage hierarchy along with an appropriate charge management policy is a promising approach to improve the salient characteristics of the EES system and reduce its amortized cost. Because the SOCs, terminal voltages, and power ratings of different EES elements may not be compatible with each other, direct connection among EES elements is not generally possible. Note that even if the EES elements have the same nominal terminal voltage, their actual voltage may vary significantly depending on their SOC.

The topology of the proposed HEES system is a flat structure because this shortens the charge transfer paths, and thus reduces the losses for charge allocation, migration and replacement. Although the physical topology is flat, the logical structure of the proposed HEES system is hierarchical. Conceptually, the architecture is closer to scratch pad rather than a cache memory in that i) the cache memory requires only a replacement policy while scratch memory requires both allocation and replacement policies, and ii) cache memory has physical hierarchy whereas scratch pad memory has a flat physical structure. Notice that the allocation, migration and replacement operations in the HEES system result in charge (content in the EES element) loss as well as timing penalty, but unlike memory hierarchy they do not results in data (content in the memory device) loss.

The charge migration path in the proposed HEES system is drawn as a single path in Figure 1, but this could in fact be either a power bus or a crossbar network. A power bus makes the migration path simpler while it allows simultaneous charge migration operations for multiple EES elements. However, the converters that participate in the charge migration process must have the same voltage level, which may prevent us from setting the optimal voltage level for the charge migration, which is a function of the SOC of the source and destination EES elements. In contrast a crossbar network enables setting of different "optimal" voltages for multiple sources and destinations at the expense of higher system cost and design complexity.

While the proposed EES system architecture is a highly flexible architecture that allows any combination of charge allocation, migration and replacement policies, we propose reconfigurability in the HEES system for enhanced cycle efficiency. The system is comprised of different banks. Each bank consists of homogeneous EES elements that meet certain voltage, power and capacity requirements. The bank is simply an array of EES elements where cell balancing is one of the most important constraints to meet. Charging/discharging efficiency is a strong function of the SOC as well as source and load voltage and current levels. Therefore, a fixed array structure cannot always guarantee desirable cycle efficiency. For example, a supercapacitor bank may have very low terminal voltage with a low SOC. If the array structure is reconfigured to have fewer parallel connections and more series connections, the same SOC for the batteries in the bank results in a higher terminal voltage for the bank itself and in turn better cycle efficiency for the HEES system.

\subsection{Charge Allocation}

Let's consider having multiple EES banks. Whenever the EES system stores electrical energy from an external power source, a proper destination for the stored energy should be chosen. The charge allocation problem is to maximize the charging efficiency by selecting the appropriate destination banks. The charging efficiency is determined by the type and SOC of the bank, voltage and current characteristics of the external power source, the degree of MPPT (maximum power point tracking) for the power source, and so forth. Most importantly, the SOC changes as charging progresses, while voltage and current levels of the external source may themselves vary over time. In cases where the optimal charge allocation policy dictators the full charging of a single EES bank, when that bank is fully charged, the second most efficient EES bank will be selected for charging and so on. When the SOC of the destination EES has a significant change during the charge process, reconfiguration of the internal connections within the bank (seriesparallel configuration) may be desirable in order to keep the highest possible charging efficiency.

\subsection{Charge Replacement}

The EES system supplies power to the load by discharging an EES bank. Similar to the charge allocation process, charge replacement is to select the most efficient EES banks which are capable of supplying electrical energy to a given load demand. Discharging efficiency is also strongly dependent on the EES element characteristics such its rate capacity characteristics, power rating, terminal voltage of the EES, and so on. Furthermore, these characteristics vary by SOC. As in the charge allocation process, the most efficient EES bank(s) to use during the system discharge varies over time, and thus charge allocation is the process of scheduling a sequence of banks to be partially or fully discharged.

\subsection{Charge Migration}

Generally, all the EES elements are subject to self-discharge. However, the amount of self-discharge is largely dependent on the EES element itself. For instance, supercapacitors can be fully discharged without any external discharge current within a week. Longterm EES should be realized by low self-discharge EES elements, which may necessitate charge migration between various EES banks.

Sometimes, the EES system may have a very high current charge allocation and replacement demand such that only certain EES banks can service the given load. These banks may however have high self-discharge rates and thus after the discharge process, the remaining charge in the high-leakage bank may be transferred to lowleakage banks for long-term storage. Conversely, when providing charge to a high-demanding load, it may be better to first transfer the charge from low-leakage, but low power rate, EES bank to a high-leakage, yet high-power EES bank. Therefore, after charge allocation or before charge replacement, charge migration may be required. Charge migration is an expensive process because of charge loss during the discharge and recharge processes. Charge migration should thus be carefully performed by accurately predicting the future load demand as well as energy possibly supplied by the external power source.

\subsection{Joint Charge Optimization}

Charge allocation, replacement and migration are complicated tasks that should consider many factors. Unfortunately, they cannot be optimized separately because of their sometime conflicting natures as well as the strong couplings among them. For example, the optimal charge allocation does not guarantee the optimal charge storage and/or charge disbursement; in fact charge migration may become inefficient in a system designed for optimal charge allocation. The joint optimization of allocation, replacement and migration is a challenging, yet critical, problem.

\section{HEES OPTIMIZATION}

We show the idea and benefits of the HEES with a simple example. Consider an EES system that performs peak shaving for a varying load. The power demand variation is repeated periodically every 7 days. The amount of energy stored to and restored from the EES is different each day: $600 \mathrm{kWh}$ for two days and $75 \mathrm{kWh}$ for the remaining five days. We assume that the amount of energy 


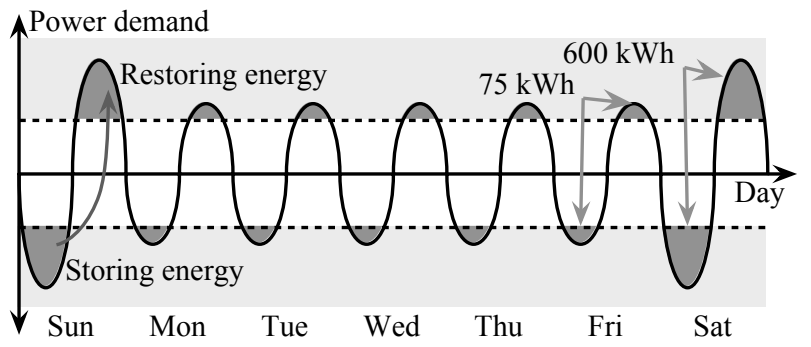

Figure 2: Power demand fluctuation example.

Table 2: Comparison between two candidate batteries for the HEES system.

\begin{tabular}{|c||c|c|}
\hline Type & Lead-acid battery & Li-ion battery \\
\hline \hline Product & Panasonic LC-X1265P & LFP-P200Ah \\
\hline Energy capacity & $780 \mathrm{Wh}$ & $640 \mathrm{Wh}$ \\
\hline Estimate price & $\sim \$ 100$ & $\sim \$ 360$ \\
\hline Cost per energy & $128.2 \$ / \mathrm{kWh}$ & $562.5 \$ / \mathrm{kWh}$ \\
\hline Cycle life & 250 & 3,000 \\
\hline
\end{tabular}

stored and restored each day are balanced, i.e., all the energy stored in a day is consumed on the same day. This is illustrated in Figure 2. For illustrative purposes only, we assume that the cycle life degradation is proportional to DoD. For example, if we use the Lead-acid battery with DoD of $50 \%$, it can be used for $250 / 0.5=500$ cycles.

Consider two cases of building an EES system i) using only Lead-acid batteries and ii) using a hybrid of Lead-acid batteries and Li-ion batteries. Table 2 is a comparison between two types of batteries under consideration in this example. Building an EES system of $600 \mathrm{kWh}$ capacity with the Lead-acid batteries only or with $\mathrm{Li}$-ion batteries only results in a total capital cost of $\$ 76,926$ and $\$ 337,500$, respectively. Obviously, the Lead-acid battery is the more cost-effective choice, and it may appear unwise to use the Li-ion battery until we consider the lifecycle cost of the two EES systems and take a longer term view of the power demand profile.

We perform a simulation on the both EES system for estimating the life cycle and amortized cost. We assume that some wearout control mechanisms, e.g., round-robin selection for discharge, are used, and so all the batteries can be replaced at the same time when them come to the end of their useful life. The lifetime and amortized cost of a Lead-acid only homogeneous EES system are 666 days and 115.5 \$/day, respectively, while those for the Li-ion only homogeneous EES system are 8,001 days and 42.2 \$/day, respectively. This clearly shows that the use of Li-ion batteries help reduce the amortized cost. However, the high capital cost prohibits us from using only Li-ion batteries.

A HEES is a good way to achieve moderate amortized cost as well as a reasonable capital cost while serving this load profile. Consider a HEES system which is composed of $525 \mathrm{kWh}(87.5 \%)$ of Lead-acid batteries and $75 \mathrm{kWh}(12.5 \%)$ of Li-ion batteries. The Lead-acid batteries, which have a short life cycle, are used only for two days a week when the power demand variation is high, while the Li-ion batteries, which have a long life cycle, are used every day. In this case, the Lead-acid batteries and Li-ion batteries should be replaced after 875 days and 3,000 days, respectively. The initial capital cost is $\$ 109,495$, and the amortized cost is $91.0 \$$ day considering the hyper period of the different life cycles.

Practical effectiveness of the HEES could be different from this simple example because the aging model and the real cost factors may be far more complicated. However, we can clearly see the ben- efits of the HEES system in terms of capital cost and amortized cost reductions. Further research is required that adopts more accurate battery models for realistic power demand profiles for optimized design and operation of HEES systems.

\section{CONCLUSIONS AND FUTURE WORK}

We presented first approach to hybridize the design of an electrical energy storage (EES) system by using a combination of various EES elements (different batteries and supercapacitors) so as to create a storage system that has the best combination of desired features, including large energy storage, low self-discharge, long cycle life, high power rating, and low cost. The key idea is to model the architecture of the hybrid EES system after that of the scratch pad memory with appropriate policies developed and implemented for charge allocation, replacement, and migration. We showed examples of how the proposed HEES system can be used to minimize the amortized cost of an electrical storage system while providing the desired level of energy storage capacity under variable load conditions and a cap on the initial capital cost.

This is an important area for further research including, but not limited to, the design of new hybrid EES system architectures for mobile and fixed storage platforms, selection of the type and bank size of storage provided by each EES element in the system, development of effective policies for charge allocation, replacement, and migration, and applications to energy management and peak shaving.

\section{ACKNOWLEDGEMENT}

Special thanks to Donghwa Shin and Jaehyun Park in Seoul National University for providing the experimental results.

\section{REFERENCES}

[1] H. T. Odum, "Energy quality and carrying capacity of the earth," Tropical Ecology, 1976.

[2] Y. Choi, N. Chang, and T. Kim, "DC-DC converter-aware power management for low-power embedded systems," T. on CAD, 2007.

[3] Y. Kim, N. Chang, Y. Wang, and M. Pedram, "Maximum power transfer tracking for a photovoltaic-supercapacitor energy system," in ISLPED, 2010.

[4] R. M. LaFollette and D. N. Bennion, "Design fundamentals of high power density, pulsed discharge, lead-acid batteries," J. of the Electrochemical Society, 1990.

[5] C. Chiasserini and R. Rao, "A model for battery pulsed discharge with recovery effect," in $W C N C, 1999$.

[6] W. Henson, "Optimal battery/ultracapacitor storage combination," $J$. of Power Sources, 2008.

[7] H. Chen, T. N. Cong, W. Yang, C. Tan, Y. Li, and Y. Ding, "Progress in electrical energy storage system: A critical review," Progress in Natural Science, 2009.

[8] F. Simjee and P. H. Chou, "Everlast: long-life, supercapacitor-operated wireless sensor node," in ISLPED, 2006.

[9] T. B. Atwater, P. J. Cygan, and F. C. Leung, "Man portable power needs of the 21st century: I. applications for the dismounted soldier ii. enhanced capabilities through the use of hybrid power sources," $J$. of Power Sources, 2000.

[10] T. Moore and J. Douglas, "Energy storage, big opportunities on a smaller scale," EPRI J., 2006.

[11] "OEM Li-ion batteries." www.panasonic.com.

[12] www.batteriesdigest.com/metal_air.htm.

[13] C. Knowlen, A. Matick, and A. Bruckner, "High efficiency energy conversion systems for liquid nitrogen automobiles," in SAE Future Transportation Technology Conference, 1998.

[14] D. Wen, H. Chen, Y. Ding, and P. Dearman, "Liquid nitrogen injection into water: Pressure build-up and heat transfer," Cryogenics, 2006. 